\title{
Structures of Two Diastereomeric Aldol Products from a New Silicon-Directed Condensation
}

\section{Citation}

Schaefer, William P., Katherine L. Widdowson, and Andrew G. Myers. 1991. Structures of two diastereomeric aldol products from a new silicon-directed condensation. Acta Crystallographica Section C Crystal Structure Communications C47(12): 2575-2578.

\section{Published Version}

http://dx.doi.org/10.1107/S010827019100522X

\section{Permanent link}

http://nrs.harvard.edu/urn-3:HUL.InstRepos:3122540

\section{Terms of Use}

This article was downloaded from Harvard University's DASH repository, and is made available under the terms and conditions applicable to Other Posted Material, as set forth at http:// nrs.harvard.edu/urn-3:HUL.InstRepos:dash.current.terms-of-use\#LAA

\section{Share Your Story}

The Harvard community has made this article openly available.

Please share how this access benefits you. Submit a story.

\section{Accessibility}


des groupes morpholino varient de 1,627 à 1,662 $\AA$; on remarquera que ces valeurs sont plus grandes que celles des liaisons $\mathrm{P}-\mathrm{N}$ du cycle phosphazène $(1,574$ à $1,612 \AA$ ). Les quatre groupes morpholino sont en configuration chaise. Cette configuration est fortement déformée dans le cas du groupe morpholino défini par les atomes $\mathrm{N} 4, \mathrm{C} 1, \mathrm{C} 2, \mathrm{O} 1, \mathrm{C} 3$ et $\mathrm{C} 4$. Les groupes morpholino en configuration chaise (Fig. 1) sont signalés par Romming \& Songstrad (1979) et Sarkar \& Gupta (1980). La projection de la structure sur le plan $y 0 z$ parallèlement à $a$ (Fig. 2) permet de mettre en évidence les différentes possibilités de polymérisation. Elle est possible lorsqu'on substitue deux atomes de chlore par une diamine. Deux sortes de chaînes se développent entre les atomes de phosphore notés $\mathrm{P} 1$ et $\mathrm{P} 2$. Ces chaînes sont parallèles soit au plan $x 0 y$ soit au plan $x 0 z$. Les valeurs des dis- tances entre ces atomes de phosphore varient de 6,565 à $9,800 \AA$. Le développement de ce réseau dans l'espace conduit à un polymère tri-dimensionnel.

\section{Références}

Allcock, H. R., Greigger, P. P., Wagner, L. J. \& Bernheim, M. Y. (1981). Inorg. Chem. 20, 716-722.

Mukhina, L. E. \& Kropacheva, A. A. (1966). J. Gen. Chem. USSR, 38, 314-317.

Romming, C. \& Songstrad, J. (1979). Acta. Chem. Scand. Ser. A, 33(3), 187-197.

Ruth, J. S., Parvez, M. \& Allcock, H. R. (1986). J. Am. Chem. Soc. 108, 6089-6090.

Sarkar, P. B. \& Gupta, S. P. (1980). Cryst. Struct. Commun. 9(4), 925-932.

SheldRICK, G. M. (1976). Program for crystal structure determination. Univ. de Cambridge, Angleterre.

Walker, N. \& Stuart, D. (1983). Acta. Cryst. A39, 158-166.

Acta Cryst. (1991). C47, 2575-2578

\title{
Structures of Two Diastereomeric Aldol Products from a New Silicon-Directed Condensation
}

\author{
By William P. Schaefer, Katherine L. Widdowson and Andrew G. Myers \\ The Beckman Institute and the Division of Chemistry and Chemical Engineering, ${ }^{*}$ \\ California Institute of Technology, Pasadena, California 91125, USA
}

(Received 18 October 1990; accepted 30 April 1991)

\begin{abstract}
Hexahydro-3,3,6-trimethyl-5-phenyl-1 $\mathrm{H}, 7 \mathrm{H}$ pyrrolo[2,1-e $][1,3,6,2]$ dioxazasilonin-7-one, $\mathrm{C}_{17} \mathrm{H}_{25}$ $\mathrm{NO}_{3} \mathrm{Si}, M_{r}=319 \cdot 48$. (2S,3R)-anti-Diastereomer (I), orthorhombic, $P 2_{1} 2_{1} 2_{1}, a=8 \cdot 716(4), b=11.677$ (3), $c=17 \cdot 161(6) \AA, \quad V=1746(1) \AA^{3}, \quad Z=4, \quad D_{x}=$ $1.22 \mathrm{~g} \mathrm{~cm}^{-3}, \lambda($ Mo $K \alpha)=0.71073 \AA$, $\mu=1.50 \mathrm{~cm}^{-1}$, $F(000)=688$, room temperature, final $R=0.040$ for 1683 reflections with $F_{o}^{2}>0 . \quad(2 S, 3 S)$-synDiastereomer (II), monoclinic, $P 2_{1}, a=6.633$ (2), $b$ $=14.645(3), c=9.630$ (2) $\AA, \beta=109.96(2)^{\circ}, \quad V=$ $879 \cdot 3(4) \AA^{3}, Z=2, D_{x}=1.21 \mathrm{~g} \mathrm{~cm}^{-3}, \lambda($ Mo $K \alpha)=$ $0.71073 \AA, \mu=1.49 \mathrm{~cm}^{-1}, F(000)=344$, room temperature, final $R=0.042$ for 2537 reflections with $F_{o}^{2}$ $>0$. The compounds have a nine-membered heterocyclic ring containing the $\mathrm{Si}$ atom, the $\mathrm{N}$ atom of the pyrrolidine ring, two $\mathrm{O}$ atoms bonded to silicon and a carbonyl $\mathrm{C}$ atom. Bond distances in the molecules are normal and equivalent bonds are equal within three times their e.s.d.'s. Isomer (I) shows a disorder in the pyrrolidine ring that is absent in isomer (II).
\end{abstract}

\footnotetext{
* Contribution No. 8230 .
}

0108-2701/91/122575-04\$03.00
Introduction. The silicon-bridged nine-membered ring aldol products (I) and (II) were formed in a new silicon-directed aldol condensation reaction (Myers
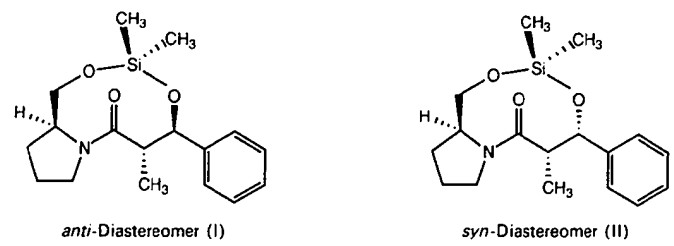

\& Widdowson, 1990). These highly crystalline products were submitted to X-ray analysis to determine stereochemical assignments and to gain insight into the mechanism of the aldol addition reaction which forms them. The structures determined herein lend credence to the notion that (I) and (II) are formed in a reaction involving pentavalent silicon. In each structure, the amide carbonyl group lies over one face of the silicon-centered tetrahedron, in van der Waals contact with the Si atom. A transition state leading to this geometry that (C) 1991 International Union of Crystallography 
involves trigonal bipyramidal silicon with methyl and enol substituents in the apical positions is easy to visualize.

Experimental. Isomer (I). Crystal an irregular cube, cut to $0.31 \times 0.35 \times 0.40 \mathrm{~mm}$; CAD-4 diffractometer; $\omega$-scan; 25 reflections with $14<2 \theta<18^{\circ}$ used to calculate cell dimensions; no absorption correction made $\left(\mu r_{\max }=0.05\right) ; \quad(\sin \theta / \lambda)_{\max }=0.60 \AA^{-1} ;$ data collected for $h$ from 0 to $10, k$ from 0 to 13 and $l$ from -20 to 20 ; standard reflections $142, \overline{1} 16$ and $\overline{3} \overline{1} 4$ showed no variations greater than predicted by counting statistics. 3500 reflections measured and merged to give 1773 unique reflections; goodness of fit for merging $0.98 ; R_{\text {int }}$ for merging 0.018 for 1551 reflections with exactly two observations; all reflections used in solution and refinement, including 90 for which $F_{o}^{2}<0$. Structure solved by MULTAN (Main, Fiske, Hull, Lessinger, Germain, Declercq \& Woolfson, 1980) which gave coordinates of $\mathrm{Si}$ and 13 other atoms; remainder found by structure factordifference map calculations; refinement used $F_{o}^{2}$ values; $H$ atoms placed at calculated positions (C- $\mathrm{H}, 0.95 \AA$ ) and repositioned once, but not refined; final refinement included coordinates and anisotropic thermal parameters of all heavy atoms (except disordered $\mathrm{C} 12 A B$, isotropic), scale factor, secondary-extinction parameters and population parameter for $\mathrm{C} 12 A(\mathrm{C} 12 B$ population $=1-\mathrm{C} 12 A)$. For all 1683 reflections with $F_{o}^{2}>0, R=0.040, w R=$ 0.006 ; for 1536 reflections with $F_{o}^{2}>3 \sigma\left(F_{o}^{2}\right), R=$ $0.035, w R=0.005, S=2.21$ for 1773 reflections and 200 parameters; variances of the individual reflections assigned based on counting statistics plus an additional term, $(0.014 l)^{2}$; variances of the merged reflections determined by standard propagation of error plus another additional term $\left(0.014 \bar{I}^{2} ; w=\right.$ $1 /\left[\sigma^{2}\left(F_{0}^{2}\right)\right]$; max. shift/e.s.d. in final least-squares cycle, 0.10 for $\mathrm{C} 12 A B, 0.01$ for all other parameters; $\max$. and min. heights in final difference Fourier map +0.25 and $-0.19 \mathrm{e} \AA^{-3}$; secondary-extinction parameter (Larson, 1967) refined to $8.7(3) \times 10^{-6}$. Atomic scattering factors taken from Cromer \& Waber (1974); dispersion corrections from Cromer (1974); programs used were those of the CRYM crystallographic computing system (Duchamp, 1964), MULTAN (Main et al., 1980) and ORTEP (Johnson, 1976). The population parameter for $\mathrm{C} 12 A$ refined to 0.45 (3), leaving $\mathrm{C} 12 B$ at 0.55 .

Isomer (II). Crystal a roughly cubic chunk, $0.44 \times$ $0.50 \times 0.41 \mathrm{~mm}$; CAD-4 diffractometer; $\omega$-scans; 25 reflections with $13<2 \theta<20^{\circ}$ used to calculate cell dimensions; no absorption correction made $\left(\mu r_{\max }=\right.$ $0.06) ;(\sin \theta / \lambda)_{\max }=0.70 \AA^{-1}$; data collected for $h$ from -9 to $9, k$ from -20 to 20 and $l$ from 0 to13; standard reflections $0 \overline{2} \overline{3}, \overline{1} 42$ and $\overline{1} \overline{5} 0$ showed a slight $(<0.5 \%)$ linear decay. 5485 reflections measured and
Table 1. Final atomic coordinates $\left(\times 10^{4}\right)$ and $U_{\text {eq }}$ values $\left(\AA^{2} \times 10^{4}\right)$ for $\mathrm{C}_{17} \mathrm{H}_{25} \mathrm{NO}_{3} \mathrm{Si}$

\begin{tabular}{lcccc}
\multicolumn{5}{c}{$U_{\text {eq }}=(1 / 3) \sum_{i} \sum_{j} U_{i j} a_{i}{ }^{*} a_{j}{ }^{*} \mathbf{a}_{i} \cdot \mathbf{a}_{j} \cdot$} \\
Isomer (I) & $x$ & $y$ & $z$ & $U_{\text {cq }}$ or $B$ \\
$\mathrm{Si}$ & $517(1)$ & $3044(1)$ & $2935(0 \cdot 4)$ & $494(2)$ \\
$\mathrm{O} 1$ & $761(2)$ & $4208(2)$ & $3457(1)$ & $459(4)$ \\
$\mathrm{O} 2$ & $-1282(2)$ & $2823(2)$ & $2703(1)$ & $595(5)$ \\
$\mathrm{C} 16$ & $1291(4)$ & $1791(3)$ & $3448(2)$ & $732(9)$ \\
$\mathrm{C} 17$ & $1432(4)$ & $3355(3)$ & $1995(2)$ & $741(9)$ \\
$\mathrm{C} 1$ & $1056(3)$ & $4365(2)$ & $4273(1)$ & $439(6)$ \\
$\mathrm{C} 2$ & $2298(3)$ & $5234(2)$ & $4378(1)$ & $434(6)$ \\
$\mathrm{C} 3$ & $3522(4)$ & $5020(3)$ & $4866(2)$ & $572(8)$ \\
$\mathrm{C} 4$ & $4630(4)$ & $5846(3)$ & $4996(2)$ & $703(9)$ \\
$\mathrm{C} 5$ & $4526(5)$ & $6882(3)$ & $4636(2)$ & $750(9)$ \\
$\mathrm{C6}$ & $3330(4)$ & $7100(3)$ & $4135(2)$ & $735(9)$ \\
$\mathrm{C} 7$ & $2226(4)$ & $6280(2)$ & $4004(2)$ & $572(7)$ \\
$\mathrm{C} 8$ & $-447(4)$ & $4737(2)$ & $4683(2)$ & $470(7)$ \\
$\mathrm{C} 9$ & $-244(4)$ & $4824(3)$ & $5561(2)$ & $632(8)$ \\
$\mathrm{C} 10$ & $-1655(4)$ & $3853(2)$ & $4476(2)$ & $490(7)$ \\
O3 & $-1645(3)$ & $2913(2)$ & $4804(1)$ & $671(6)$ \\
$\mathrm{N}$ & $-2631(3)$ & $4085(2)$ & $3890(1)$ & $556(6)$ \\
$\mathrm{C} 11$ & $-2739(4)$ & $5157(3)$ & $3450(2)$ & $663(8)$ \\
$\mathrm{C} 12 A$ & $-3721(14)$ & $4689(13)$ & $2726(8)$ & $5.9(4) *$ \\
$\mathrm{C} 12 B$ & $-4115(14)$ & $5086(12)$ & $2973(8)$ & $7 \cdot 1(3)^{*}$ \\
$\mathrm{C} 13$ & $-4514(5)$ & $3779(4)$ & $2945(3)$ & $1150(15)$ \\
$\mathrm{C} 14$ & $-3504(4)$ & $3167(3)$ & $3529(2)$ & $758(9)$ \\
$\mathrm{C} 15$ & $-2431(4)$ & $2281(3)$ & $3164(2)$ & $707(9)$ \\
& & & &
\end{tabular}

Isomer (II)

$\begin{array}{lllll}\mathrm{Si} & 8618(0.9) & 4053 & 1548(0 \cdot 6) & 451(1) \\ \mathrm{O} 1 & 8309(2) & 2961(1) & 1809(2) & 468(3) \\ \mathrm{O} 2 & 7386(3) & 4371(1) & -160(2) & 561(4) \\ \mathrm{C} 16 & 11546(4) & 4199(2) & 2122(3) & 608(6) \\ \mathrm{C} 17 & 7415(5) & 4758(2) & 2640(3) & 722(7) \\ \mathrm{Cl} & 6304(3) & 2496(2) & 1413(2) & 419(4) \\ \mathrm{C} 2 & 5941(3) & 2106(2) & 2758(2) & 431(4) \\ \mathrm{C} 3 & 7598(4) & 2010(2) & 4095(3) & 547(5) \\ \mathrm{C} 4 & 7240(5) & 1638(2) & 5307(3) & 648(7) \\ \mathrm{C} 5 & 5220(5) & 1361(2) & 5202(3) & 658(7) \\ \mathrm{C} 6 & 3562(5) & 1447(2) & 3891(3) & 662(6) \\ \mathrm{C} 7 & 3903(4) & 1827(2) & 2664(3) & 544(5) \\ \mathrm{C} 8 & 6271(4) & 1749(2) & 261(3) & 469(5) \\ \mathrm{C} 9 & 7737(5) & 953(2) & 914(3) & 647(6) \\ \mathrm{C} 10 & 6916(4) & 2226(2) & -924(2) & 473(5) \\ \mathrm{O} 3 & 8714(3) & 2133(2) & -991(2) & 662(4) \\ \mathrm{N} & 5526(3) & 2837(1) & -1787(2) & 492(4) \\ \mathrm{C} 11 & 3231(4) & 2916(3) & -2060(3) & 797(8) \\ \mathrm{C} 12 & 2461(5) & 3483(3) & -3452(4) & 904(9) \\ \mathrm{C} 13 & 4271(5) & 4120(2) & -3315(3) & 811(8) \\ \mathrm{C} 14 & 6292(4) & 3578(2) & -2514(3) & 558(6) \\ \mathrm{C} 15 & 8020(4) & 4133(2) & -1393(3) & 610(6)\end{array}$

* Isotropic displacement parameter, $B\left(\AA^{2}\right)$.

merged to give 2661 unique reflections; goodness of fit for merging 1.02; $R_{\text {int }}$ for merging 0.019 for 2200 reflections with exactly two observations; all reflections used in solution and refinement, including 124 for which $F_{o}^{2}<0$. Structure solved by MULTAN (Main et al., 1980) which gave the coordinates of $\mathrm{Si}$ and nine other atoms, the remainder being located by structure factor-Fourier synthesis calculations; refinement used $F_{o}^{2}$ values; $\mathrm{H}$ atoms were placed at calculated positions $(\mathrm{C}-\mathrm{H}=0.95 \AA)$ assuming staggered geometry and repositioned once but not refined; final refinement included coordinates and anisotropic thermal parameters for all heavy atoms ( $y$ coordinate of $\mathrm{Si}$ held constant to fix the origin) and a scale factor. For all 2537 reflections with $F_{o}^{2}>$ $0, R=0.042$ and $w R=0.007$; for 2239 reflections with $F_{o}^{2}>3 \sigma\left(F_{o}^{2}\right), R=0.036$ and $w R=0.006 ; S=$ 
Table 2. Distances $(\AA)$ and angles $\left(^{\circ}\right)$ not involving $\mathrm{H}$ atoms for $\mathrm{C}_{17} \mathrm{H}_{25} \mathrm{NO}_{3} \mathrm{Si}$

$\begin{array}{ll}\text { Isomer (I) } & \\ \mathrm{Si}-\mathrm{O} 1 & 1.642(2) \\ \mathrm{Si}-\mathrm{O} 2 & 1.638(2) \\ \mathrm{Si}-\mathrm{C} 16 & 1.837(4) \\ \mathrm{Si}-\mathrm{C} 17 & 1.836(3) \\ \mathrm{O} 1-\mathrm{C} 1 & 1.435(3) \\ \mathrm{O} 2-\mathrm{C} 15 & 1.425(4) \\ \mathrm{C} 1-\mathrm{C} 2 & 1.495(4) \\ \mathrm{C}-\mathrm{C} 8 & 1.549(4) \\ \mathrm{C} 2-\mathrm{C} 3 & 1.380(4) \\ \mathrm{C} 2-\mathrm{C} 7 & 1.381(4) \\ \mathrm{C} 3-\mathrm{C} 4 & 1.382(5) \\ \mathrm{C} 4-\mathrm{C} 5 & 1.362(5) \\ \mathrm{C} 5-\mathrm{C} 6 & 1.374(5) \\ \mathrm{C} 6-\mathrm{C} 7 & 1.376(5)\end{array}$

\begin{tabular}{|c|c|c|c|}
\hline $\mathrm{O} 2-\mathrm{Si}-\mathrm{O} 1$ & $112 \cdot 8(1)$ & $\mathrm{C} 9-\mathrm{C} 8-\mathrm{Cl}$ & $111.8(2)$ \\
\hline $\mathrm{Cl} 6-\mathrm{Si}-\mathrm{Ol}$ & $110.5(1)$ & $\mathrm{Cl}-\mathrm{C} 8-\mathrm{Cl}$ & $106.8(2)$ \\
\hline $\mathrm{C} 17-\mathrm{Si}-\mathrm{Ol}$ & $105 \cdot 1(1)$ & $\mathrm{Cl} 0-\mathrm{C} 8-\mathrm{C} 9$ & $111 \cdot 0(2)$ \\
\hline $\mathrm{Cl} 6-\mathrm{Si}-\mathrm{O} 2$ & $110.0(1)$ & $\mathrm{O} 3-\mathrm{Cl} 10-\mathrm{C} 8$ & $119 \cdot 6(3)$ \\
\hline $\mathrm{Cl} 7-\mathrm{Si}-\mathrm{O} 2$ & $103.5(1)$ & $\mathrm{N}-\mathrm{Cl}-\mathrm{C} 8$ & $118.5(2)$ \\
\hline $\mathrm{Cl} 7-\mathrm{Si}-\mathrm{Cl} 6$ & $114.8(2)$ & $\mathrm{N}-\mathrm{Cl}-\mathrm{O} 3$ & $121 \cdot 6(3)$ \\
\hline $\mathrm{Cl}-\mathrm{O} 1-\mathrm{Si}$ & $131.4(2)$ & $\mathrm{C} 11-\mathrm{N}-\mathrm{C} 10$ & $126.8(2)$ \\
\hline $\mathrm{Cl} 15-\mathrm{O} 2-\mathrm{Si}$ & $127.4(2)$ & $\mathrm{Cl} 4-\mathrm{N}-\mathrm{ClO}$ & $120 \cdot 1(3)$ \\
\hline $\mathrm{C} 2-\mathrm{C} 1-\mathrm{Ol}$ & $109.5(2)$ & $\mathrm{Cl} 4-\mathrm{N}-\mathrm{Cll}$ & $112 \cdot 1(2)$ \\
\hline $\mathrm{C} 8-\mathrm{Cl}-\mathrm{O} 1$ & $109 \cdot 1(2)$ & $\mathrm{C} 12 A-\mathrm{C} 11-\mathrm{N}$ & $98 \cdot 2(5)$ \\
\hline $\mathrm{C} 8-\mathrm{C} 1-\mathrm{C} 2$ & $111 \cdot 6(2)$ & $\mathrm{Cl} 2 \mathrm{~B}-\mathrm{ClI}-\mathrm{N}$ & $107 \cdot 1(6)$ \\
\hline $\mathrm{C} 3-\mathrm{C} 2-\mathrm{Cl}$ & $120 \cdot 7(2)$ & $\mathrm{Cl} 3-\mathrm{Cl} 2 A-\mathrm{Cl}$ & $109 \cdot 5(9)$ \\
\hline $\mathrm{C} 7-\mathrm{C} 2-\mathrm{Cl}$ & $120 \cdot 8(2)$ & $\mathrm{Cl} 3-\mathrm{C} 12 \mathrm{~B}-\mathrm{C} 11$ & $104.8(8)$ \\
\hline $\mathrm{C} 7-\mathrm{C} 2-\mathrm{C} 3$ & $118 \cdot 5(3)$ & $\mathrm{C} 14-\mathrm{Cl} 3-\mathrm{C} 12 \mathrm{~A}$ & $105 \cdot 3(7)$ \\
\hline $\mathrm{C} 4-\mathrm{C} 3-\mathrm{C} 2$ & $120 \cdot 8(3)$ & $\mathrm{Cl} 4-\mathrm{Cl} 3-\mathrm{Cl} 2 \mathrm{~B}$ & $108 \cdot 1(6)$ \\
\hline $\mathrm{C} 5-\mathrm{C} 4-\mathrm{C} 3$ & $120 \cdot 0(3)$ & $\mathrm{C} 13-\mathrm{C} 14-\mathrm{N}$ & $103.8(3)$ \\
\hline $\mathrm{C} 6-\mathrm{C} 5-\mathrm{C} 4$ & $119.9(3)$ & $\mathrm{Cl} 5-\mathrm{Cl} 4-\mathrm{N}$ & $110 \cdot 7(3)$ \\
\hline $\mathrm{C} 7-\mathrm{C} 6-\mathrm{C} 5$ & $120 \cdot 2(3)$ & $\mathrm{Cl} 5-\mathrm{Cl} 4-\mathrm{Cl} 3$ & $113.9(3)$ \\
\hline $\mathrm{C} 6-\mathrm{C} 7-\mathrm{C} 2$ & $120.5(3)$ & $\mathrm{C} 14-\mathrm{Cl} 5-\mathrm{O} 2$ & $110.9(3)$ \\
\hline
\end{tabular}

Isomer (II)

$\begin{array}{ll}\mathrm{Si}-\mathrm{O} 1 & 1.643(2) \\ \mathrm{Si}-\mathrm{O} 2 & 1.635(2) \\ \mathrm{Si}-\mathrm{Cl} 16 & 1.841(3) \\ \mathrm{Si}-\mathrm{C} 17 & 1.839(3) \\ \mathrm{O} 1-\mathrm{Cl} & 1.425(3) \\ \mathrm{O} 2-\mathrm{C} 15 & 1.432(3) \\ \mathrm{C} 1-\mathrm{C} 2 & 1.508(3) \\ \mathrm{C} 1-\mathrm{C} 8 & 1.553(3) \\ \mathrm{C} 2-\mathrm{C} 3 & 1.385(3) \\ \mathrm{C} 2-\mathrm{C} 7 & 1.385(3) \\ \mathrm{C} 3-\mathrm{C} 4 & 1.380(4) \\ \mathrm{C} 4-\mathrm{C} 5 & 1.370(4)\end{array}$

$\mathrm{O} 2-\mathrm{Si}-\mathrm{O} 1$

$\mathrm{C} 16-\mathrm{Si}-\mathrm{O} 1$

$\mathrm{Cl} 7-\mathrm{Si}-\mathrm{O} 1$

$\mathrm{Cl} 6-\mathrm{Si}-\mathrm{O} 2$

$\mathrm{Cl} 7-\mathrm{Si}-\mathrm{O} 2$

$\mathrm{C} 17-\mathrm{Si}-\mathrm{Cl} 6$

$\mathrm{Cl}-\mathrm{Ol}-\mathrm{Si}$

$\mathrm{C} 15-\mathrm{O} 2-\mathrm{Si}$

$\mathrm{C} 2-\mathrm{Cl}-\mathrm{OI}$

$\mathrm{C} 8-\mathrm{Cl}-\mathrm{Ol}$

$\mathrm{C} 8-\mathrm{Cl}-\mathrm{C} 2$

$\mathrm{C} 3-\mathrm{C} 2-\mathrm{C} 1$

$\mathrm{C} 7-\mathrm{C} 2-\mathrm{Cl}$

$\mathrm{C} 7-\mathrm{C} 2-\mathrm{C} 3$

$\mathrm{C} 4-\mathrm{C} 3-\mathrm{C} 2$

$\mathrm{C} 5-\mathrm{C} 4-\mathrm{C} 3$

$\mathrm{C} 6-\mathrm{C} 5-\mathrm{C} 4$
$\mathrm{C} 7-\mathrm{C} 6-\mathrm{C} 5$

2.32 for 2661 reflections and 198 parameters; weights calculated as for isomer (I); max. shift/e.s.d. in final least-squares cycle $<0.005$; max. and min. heights in the final difference map $+0 \cdot 22$ and $-0 \cdot 17$ e $\AA^{-3}$; no apparent need for secondary-extinction correction; scattering factors and computer programs as for isomer (I).

$\begin{array}{ll}\mathrm{C} 5-\mathrm{C} 6 & 1.368(4) \\ \mathrm{C} 6-\mathrm{C} 7 & 1.392(4) \\ \mathrm{C} 8-\mathrm{C} 9 & 1.511(4) \\ \mathrm{C} 8-\mathrm{C} 10 & 1.518(3) \\ \mathrm{C} 10-\mathrm{O} 3 & 1.224(3) \\ \mathrm{C} 10-\mathrm{N} & 1.348(3) \\ \mathrm{N}-\mathrm{C} 11 & 1.459(4) \\ \mathrm{N}-\mathrm{C} 14 & 1.473(3) \\ \mathrm{C} 11-\mathrm{C} 12 & 1.510(5) \\ \mathrm{C} 12-\mathrm{C} 13 & 1.490(5) \\ \mathrm{C} 13-\mathrm{C} 14 & 1.522(4) \\ \mathrm{C} 14-\mathrm{C} 15 & 1.515(4)\end{array}$

$\begin{array}{lll}112.7(1) & \mathrm{C} 6-\mathrm{C} 7-\mathrm{C} 2 & 120.0(2) \\ 103.9(1) & \mathrm{C} 9-\mathrm{C} 8-\mathrm{Cl} & 113.3(2) \\ 111.0(1) & \mathrm{C} 10-\mathrm{C} 8-\mathrm{Cl} & 105.9(2) \\ 112.5(1) & \mathrm{C} 10-\mathrm{C} 8-\mathrm{C} 9 & 111.2(2) \\ 104.5(1) & \mathrm{O} 3-\mathrm{C} 10-\mathrm{C} 8 & 121.2(2) \\ 112.5(1) & \mathrm{N}-\mathrm{C} 10-\mathrm{C} 8 & 117.4(2) \\ 125.3(1) & \mathrm{N}-\mathrm{C} 10-\mathrm{O} 3 & 121.0(2) \\ 125.3(2) & \mathrm{C} 11-\mathrm{N}-\mathrm{C} 10 & 128.0(2) \\ 111.1(2) & \mathrm{C} 14-\mathrm{N}-\mathrm{C} 10 & 120.5(2) \\ 108.2(2) & \mathrm{C} 14-\mathrm{N}-\mathrm{C} 11 & 111.3(2) \\ 112.3(2) & \mathrm{C} 12-\mathrm{C} 11-\mathrm{N} & 102.7(3) \\ 121.9(2) & \mathrm{C} 13-\mathrm{C} 12-\mathrm{C} 11 & 104.3(3) \\ 119.6(2) & \mathrm{C} 14-\mathrm{C} 13-\mathrm{C} 12 & 105.2(3) \\ 118.5(2) & \mathrm{C} 13-\mathrm{C} 14-\mathrm{N} & 103.8(2) \\ 121.0(2) & \mathrm{C} 15-\mathrm{C} 14-\mathrm{N} & 110.9(2) \\ 120.0(3) & \mathrm{C} 15-\mathrm{C} 14-\mathrm{C} 13 & 113.8(2) \\ 119.9(3) & \mathrm{C} 14-\mathrm{C} 15-\mathrm{O} 2 & 110.9(2) \\ 120.5(3) & & \end{array}$

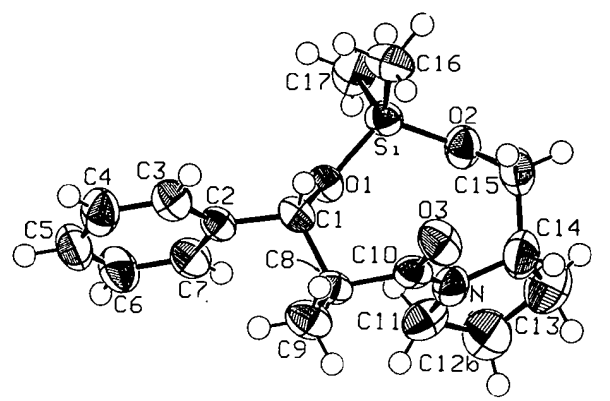

(a)

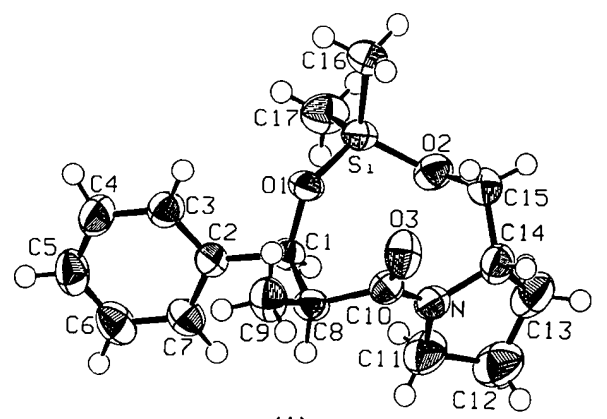

(b)

Fig. 1. An ORTEP drawing of (a) isomer (I) and (b) isomer (II) with $50 \%$ thermal ellipsoids. $\mathrm{H}$ atoms are shown as open spheres of arbitrary (small) dimension. (Atom C12 $A$ is not shown.)

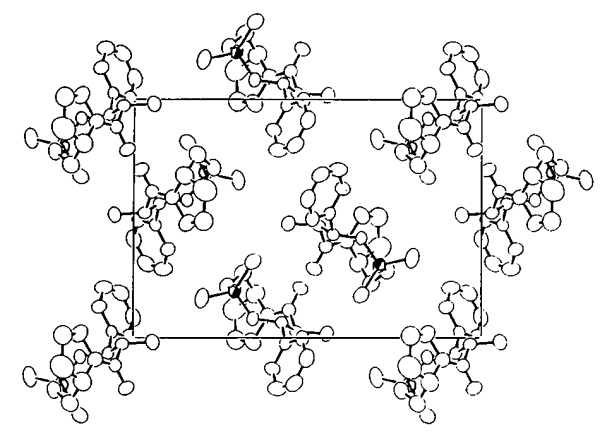

Fig. 2. An $O R T E P$ projection of isomer (I), down the $a$ axis; the $c$ axis is vertical. A unit cell is outlined, the $\mathrm{Si}$ atom is shown shaded, $\mathrm{H}$ atoms are not shown, $50 \%$ thermal ellipsoids.

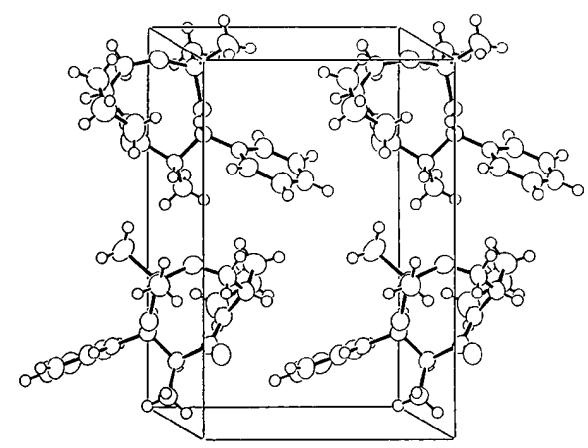

Fig. 3. An ORTEP projection of isomer (II) roughly perpendicular to the $b c$ plane. One unit cell is outlined and the contents of two unit cells are shown. Heavy atoms are shown as $50 \%$ thermal ellipsoids, $\mathrm{H}$ atoms as arbitrary (small) spheres. 
Final parameters are given in Table $1^{*}$ for isomers (I) and (II).

Discussion. The two stereoisomers whose structures are described here show good similarities in bond distances and regular structures with no significant departures from expected bond lengths or angles. The disorder at $\mathrm{C} 12$ in isomer (I) makes comparisons in that region meaningless, but for the rest of the molecule these statements hold true (Table 2). The nine-membered ring has a certain amount of flexibility, but the two conformations, while differing at $\mathrm{Cl}$, are otherwise nearly equivalent. The carbonyl bond in both isomers exhibits normal van der Waals contacts across the ring: $\mathrm{Si}$ to the center of $(\mathrm{C} 10, \mathrm{O} 3)$ is $3.56 \AA$ in isomer (I) and $3.62 \AA$ in isomer (II). We expected $3.55-3.65 \AA$ based on data taken from Pauling (1960). The shortest (relatively speaking) contact we observed is $\mathrm{Si}-\mathrm{C} 10=3.39 \AA$ in isomer (I); this is still approximately a van der Waals distance (radii for $\mathrm{Si}=1.95, \mathrm{C}=1.50 \AA$ ).

In both structures the ketone $\mathrm{O}$ atom $\mathrm{O} 3$ has some contacts with $\mathrm{H}$ atoms on the benzene ring. In isomer (I) $\mathrm{O} 3 \cdots \mathrm{H} 3=2.59 \AA$ and in isomer (II) $\mathrm{O} 3 \cdots \mathrm{H} 4=2 \cdot 71 \AA$. These are not short enough to be considered as hydrogen bonds, but may indicate a

\footnotetext{
* Lists of structure factors, anisotropic thermal parameters, complete distances and angles, and $\mathrm{H}$-atom parameters have been deposited with the British Library Document Supply Centre as Supplementary Publication No. SUP 54215 (31 pp.). Copies may be obtained through The Technical Editor, International Union of Crystallography, 5 Abbey Square, Chester CH1 2HU, England.
}

small interaction between molecules in the crystal. Fig. 1 shows the numbering schemes for isomers (I) and (II). Figs. 2 and 3 show the packing of the isomers (I) and (II).

We thank the National Science Foundation, the David and Lucile Packard Foundation, and the Caltech Consortium in Chemistry and Chemical Engineering (members: E. I. duPont de Nemours \& Co., Inc., Eastman Kodak Company, and Minnesota Mining and Manufacturing Company) for financial support.

\section{References}

CROMER, D. T. (1974). International Tables for X-ray Crystallography, Vol. IV, pp. 149-151. Birmingham: Kynoch Press. (Present distributor Kluwer Academic Publishers, Dordrecht.)

Cromer, D. T. \& WABER, J. T. (1974). International Tables for X-ray Crystallography, Vol. IV, pp. 99-101. Birmingham: Kynoch Press. (Present distributor Kluwer Academic Publishers, Dordrecht.)

Duchamp, D. J. (1964). Am. Crystallogr. Assoc. Meet, Bozeman, Montana, Paper B14, p. 29.

JoHnson, C. K. (1976). ORTEPII. Report ORNL-3794. Oak Ridge National Laboratory, Oak Ridge, Tennessee, USA.

LaRson, A. C. (1967). Acta Cryst. 23, 664-665.

Main, P., Fiske, S. J., Hull, S. E., Lessinger, L., Germain, G., DeClerCQ, J.-P. \& WoOlfson, M. M. (1980). MULTAN80. A System of Computer Programs for the Automatic Solution of Crystal Structures from $X$-ray Diffraction Data. Univs. of York, England, and Louvain, Belgium.

Myers, A. G. \& Widdowson, K. L. (1990). J. Am. Chem. Soc. $112,9672-9674$.

Pauling, L. (1960). The Nature of the Chemical Bond, 3rd ed. Ithaca, NY: Cornell Univ. Press.

Acta Cryst. (1991). C47, 2578-2580

\title{
Structure of a Chiral Cyclopentanone Precursor in Neocarzinostatin Synthetic Studies, $\mathrm{C}_{20} \mathrm{H}_{21} \mathrm{NO}_{3} \mathrm{~S} . \mathrm{H}_{2} \mathrm{O}$
}

\author{
By William P. Schaffer, Elaine Y. Kuo, Philip M. Harrington and Andrew G. Myers \\ The Beckman Institute and Division of Chemistry and Chemical Engineering, ${ }^{*}$ \\ California Institute of Technology, Pasadena, California 91125, USA
}

(Received 26 October 1990; accepted 3 May 1991)

\begin{abstract}
E, 2 S, 3 R)-2-[(4 R, 5 R)-4,5-$ Dimethyl-1,3dioxolan-2-yl]-3-(2-naphthylthio)cyclopentanone oxime, $M_{r}=373.47$, orthorhombic, $P 2_{1} 2_{1} 2_{1}, a=$ $5 \cdot 374(13), \quad b=18 \cdot 135(3), \quad c=20 \cdot 487(5) \AA, \quad V=$ 1997 (5) $\AA^{3}, Z=4, D_{x}=1.24 \mathrm{~g} \mathrm{~cm}^{-3}$, Mo $K \alpha, \lambda=$ $0.71073 \AA, \mu=1.87 \mathrm{~cm}^{-1}, F(000)=792$, room tem-

* Contribution No. 8229 .
\end{abstract}

0108-2701/91/122578-03\$03.00 perature, crystal volume $2.7 \times 10^{-4} \mathrm{~mm}^{3}$, final $R=$ 0.127 for 971 reflections with $F_{o}^{2}>0$ out of 1131 data, $R=0.065$ for 467 reflections with $F_{o}^{2}>3 \sigma\left(F_{o}^{2}\right)$. Despite the small crystal size (all larger crystals were twinned), a structural solution of adequate precision was obtained. Bond distances and angles in the molecule are normal; the water molecules are hydrogen bonded in a chain along the $a$ axis and each

(c) 1991 International Union of Crystallography 